\section{PET/MR Imaging: A Case-Based Approach}

R. Gupta, R. Matthews, L. Bangiyev, D. Franceschi, and M. Schweitzer, Eds.

New York, NY: Springer, 2018, 351 pages, $\$ 179.99$

PET/MRI is an exciting novel diagnostic imaging modality that combines the precise anatomic and functional information provided by MRI with the molecular data obtained with PET. It offers the promise of a simplified workflow, reduced radiation, wholebody imaging with superior soft-tissue contrast, and time-of-flight functional information. Its clinical applications have been rapidly emerging over the last several years, and the use of this modality is bound to gain momentum in the diagnosis and treatment of various diseases.

Editors, mostly in the Department of Radiology at Stony Brook University Hospital, created this book to provide a case-based overview of PET/MRI for the novice since there is a paucity of resources that compile a collection of high-quality PET/MRI cases. Interpretation of PET/MRI requires understanding of both PET and MRI findings, and MRI, with its various sequences, is more complex to master.

The book, written by 29 contributors, is divided into 6 parts according to organ systems, covering the use of PET/MRI technique in the diagnosis of oncologic, neurologic, cardiovascular, infectious, inflammatory, and pediatric diseases. Part I has 25 musculoskeletal cases; part II, 20 chest cases; part III, 30 gastrointestinal cases; part IV, 19 genitourinary cases; part V, 18 head and neck cases; and part VI, 26 neurologic cases. Each case, with proven pathology, is presented with a brief patient history, imaging findings, and their pearls and pitfalls and is accompanied by high-quality PET/ MR images. However, there is no diagnosis or pathologic findings in the figure legends. Updated references as suggested reading are provided at the end of each case. The index is helpful.

The major strength of this book is the discussion of both MRI and PET findings pertinent to each case. The discussion of various oncologic cases highlights staging, restaging, therapeutic response, and follow-up. This book should be interesting to all radiologists and nuclear physicians who wish to learn more about PET/MRI developments and clinical applications.

\author{
E. Edmund Kim \\ University of California at Irvine \\ 101 The City Dr. S. \\ Orange, CA 92868 \\ E-mail: edmundek@uci.edu
}

